\title{
Case Report of Eustachian Tube Malignant Melanoma: 4-Year Follow-up of a Grave and Rare Condition
}

\section{Dear Editor,}

Mucosal melanoma of the Eustachian tube is extremely rare. There are only 10 other case reports of mucosal melanoma of Eustachian tube origin. Here, we share our 4-year follow-up of a 55-year-old Chinese lady with right Eustachian tube melanoma. We also review other case reports of this grave condition and discuss the management options and prognoses observed.

\section{Methods}

Our patient is a 55-year old Chinese lady. She presented with right-sided conductive hearing loss of 2 months duration. Right serous otitis media was seen on otoscopy. Nasoendoscopy showed a black mass at the right Eustachian tube opening (Figure 1). A biopsy of this mass was done and histology revealed malignant melanoma.

Magnetic Resonance Imaging (MRI) of the post nasal space showed a $11 \times 4 \mathrm{~mm}$, hypo-enhancing, T1- and T2-weighted isointense lesion at the cartilaginous portion of the right Eustachian tube, extending to its pharyngeal orifice. This caused proximal obstruction and resultant mastoid effusion. The tumour was centered in the bony Eustachian tube, with involvement of the right torus tubarius. The tumour did not appear to extend into or beyond the middle ear orifice (Figure 2). There was no cervical lymphadenopathy seen on MRI.

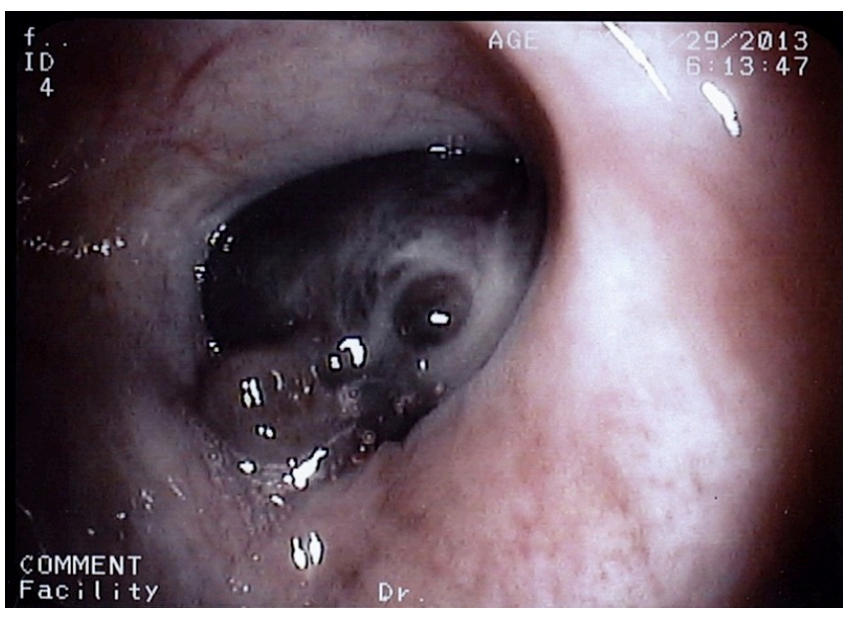

Fig. 1. Nasoendoscopy of our patient's right Eustachian tube opening with the hyperpigmented tumour.

Computed tomography (CT) scan of the temporal bone revealed soft tissue opacification of the right Eustachian tube. There was also opacification of the middle ear, mastoid antrum and air cells. There was no mass effect or bony erosion. There were no distant metastases.

Preoperatively, there was concern that the tumour was located close to the petrous part of the internal carotid artery. Hence, a balloon occlusion test was performed.

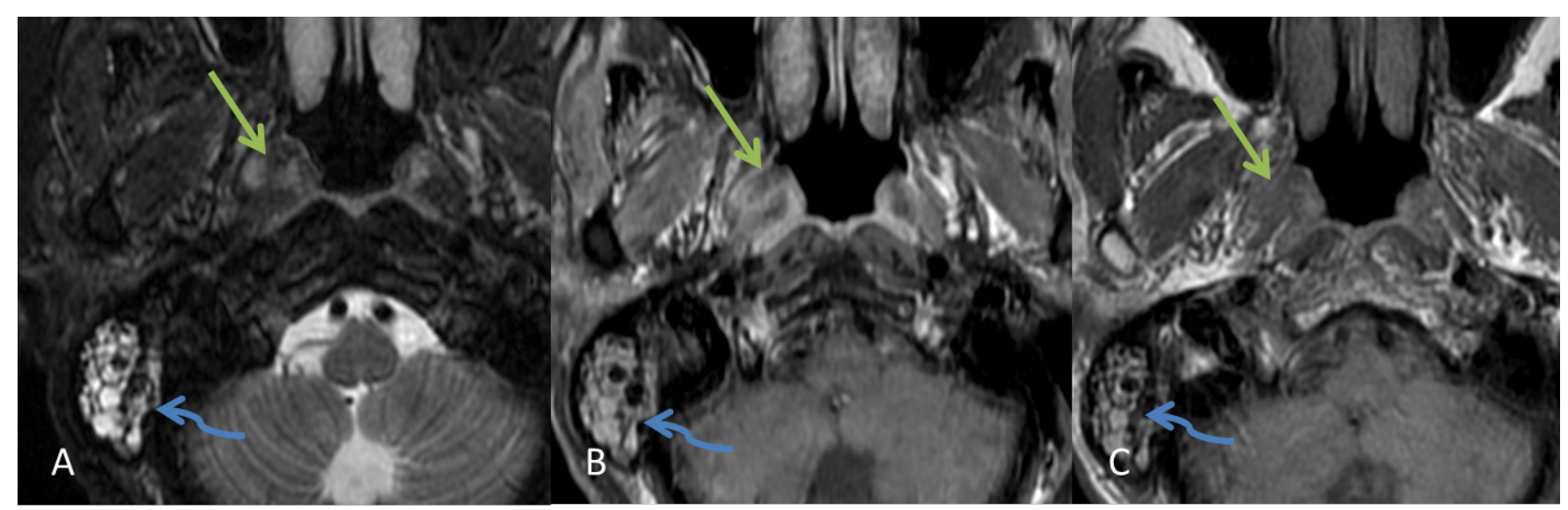

Fig. 2. Magnetic Resonance Imaging (A) Dixon, (B) T1w FS post contrast, (C) T1w pre-contrast sequences, reveals a non-enhancing 11 x 4mm, hypo-enhancing mass at the right cartilaginous Eustachian tube (Straight green arrow). It is T1w isointense. The obstruction results in right mastoid effusion (blue curvy arrow). 
During balloon occlusion test of the right internal carotid artery, there was good crossover flow from the left internal carotid artery with no significant delay in the venous return compared to the baseline. Neurological assessment performed at $0,5,10$ and 15 minutes did not reveal any focal neurological deficit.

The patient's case was discussed at our multidisciplinary tumour board and the decision was made for surgery and adjuvant radiotherapy. She underwent infratemporal fossa Fisch B approach and open nasopharyngectomy via maxillary swing with en-bloc excision of the Eustachian tube and tumour. Figure 3 shows the maxillary swing to expose the eustachian tube opening in the nasopharynx. The eustachian tube was traced from the bony to the cartilaginous portion and removed in entirety with the tumour. The petrous carotid artery was delineated. A diamond burr was used to drill in the areas of the dura and carotid artery in order to prevent injury to these delicate areas. The nasopharyngeal defect was obliterated with a temporalis muscle flap.

Histological analysis showed tumour cells positive for human melanoma black (HMB-45) and patchily positive for Melan-A. Ki-67 staining indicated a MIB-1 index of

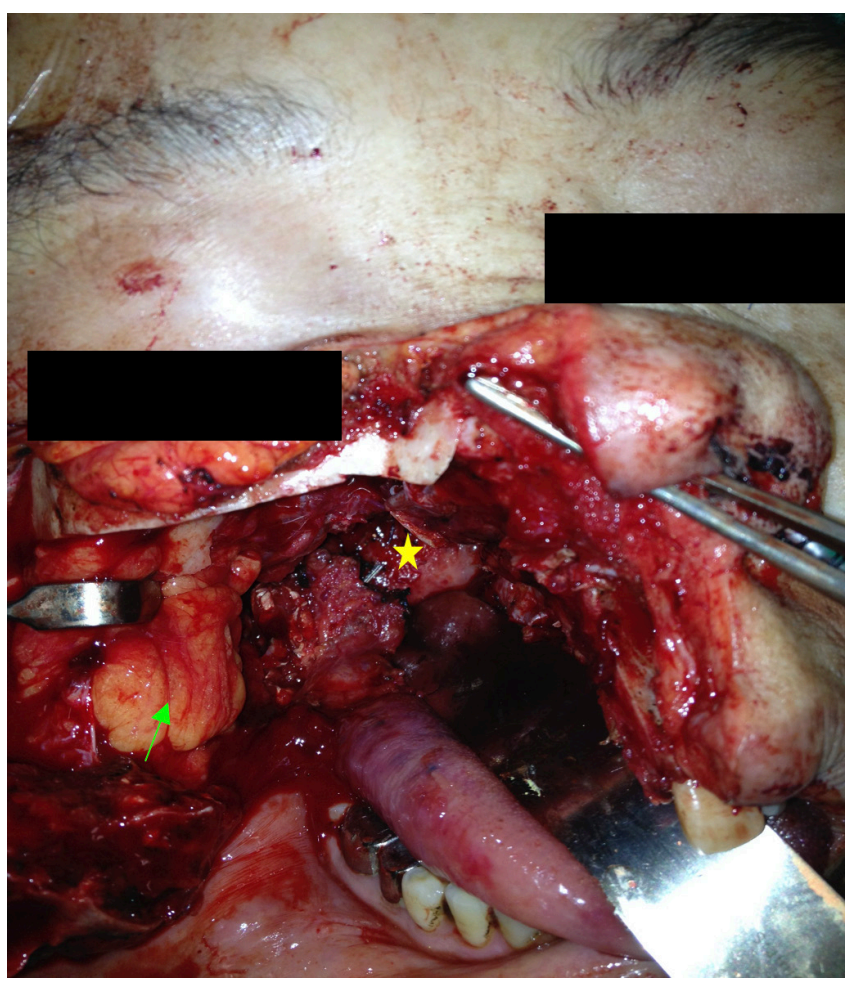

Fig. 3. Right maxillary swing to expose opening of the Eustachian tube in the nasopharynx. A clip (yellow star) has been placed over the resected area in the event radiotherapy is needed. The temporalis muscle is later used to cover the defect in the nasopharynx. Green arrow is pointing to right buccal fat. approximately $10-15 \%$ in the tumour. No lymphovascular invasion and no perineural invasion was seen. The tumour measured up to $1.5 \mathrm{~mm}$ thick. It did not invade the cartilage and was $1 \mathrm{~mm}$ from the closest radial margin. Both medial and lateral resection margins were not involved by tumour. Melanin pigments were present in the acinar and ductal cell of surrounding mucinous glands.

The patient completed 60 Gy of radiation therapy (RT) in 30 fractions using a 7-field intensity modulated radiation therapy (IMRT) plan to the tumour bed only. 1-year post-treatment CT scan did not reveal any recurrence.

\section{Results}

21 months post operatively, the patient complained of right sided neck swelling. There was a $2 \mathrm{~cm}$ right level 2 cervical lymph node on palpation. The rest of the head and neck examination was unremarkable. Fine needle aspiration of the mass confirmed malignant melanoma. Positron emission tomography-CT (PETCT) showed an enlarged hypermetabolic right level 2 cervical lymph node measuring $2.2 \times 1.5 \mathrm{~cm}$ with SUVmax 6.9, which was highly suspicious for nodal metastasis (Figure 4). A right modified radical neck dissection with preservation of the spinal accessory nerve, internal jugular vein and sternocleidomastoid muscle was performed. The final histology revealed 1 out of 28 lymph nodes positive for malignancy, with an enlarged blackish brown lymph node measuring $2.5 \mathrm{~cm}$ in maximum dimension in level 2 .

7 months post neck dissection, there was a subcutaneous $1-\mathrm{cm}$ lump noted at the right neck level 2 region. During excision under local anaesthesia, 2 lumps were noted at the same region and histology confirmed metastatic melanoma. The patient underwent wide excision under general anaesthesia. The deep margins were consistent with metastatic deposit of malignant melanoma.

The patient's case was discussed at the Head and Neck multidisciplinary tumour board. Tumour board suggested single modality treatment with chemotherapy, as repeat radiation therapy was not suitable since she had previously undergone radiation. The patient was not keen on chemotherapy. She subsequently deceased from pulmonary metastases, 4 years from the initial diagnosis of Eustachian tube melanoma.

\section{Discussion}

Mucosal melanomas are rare and constitute only $1.3 \%$ of all cutaneous and non-cutaneous melanomas. Noncutaneous mucosal melanoma is most common in the head and neck with distribution as follows: head and neck 

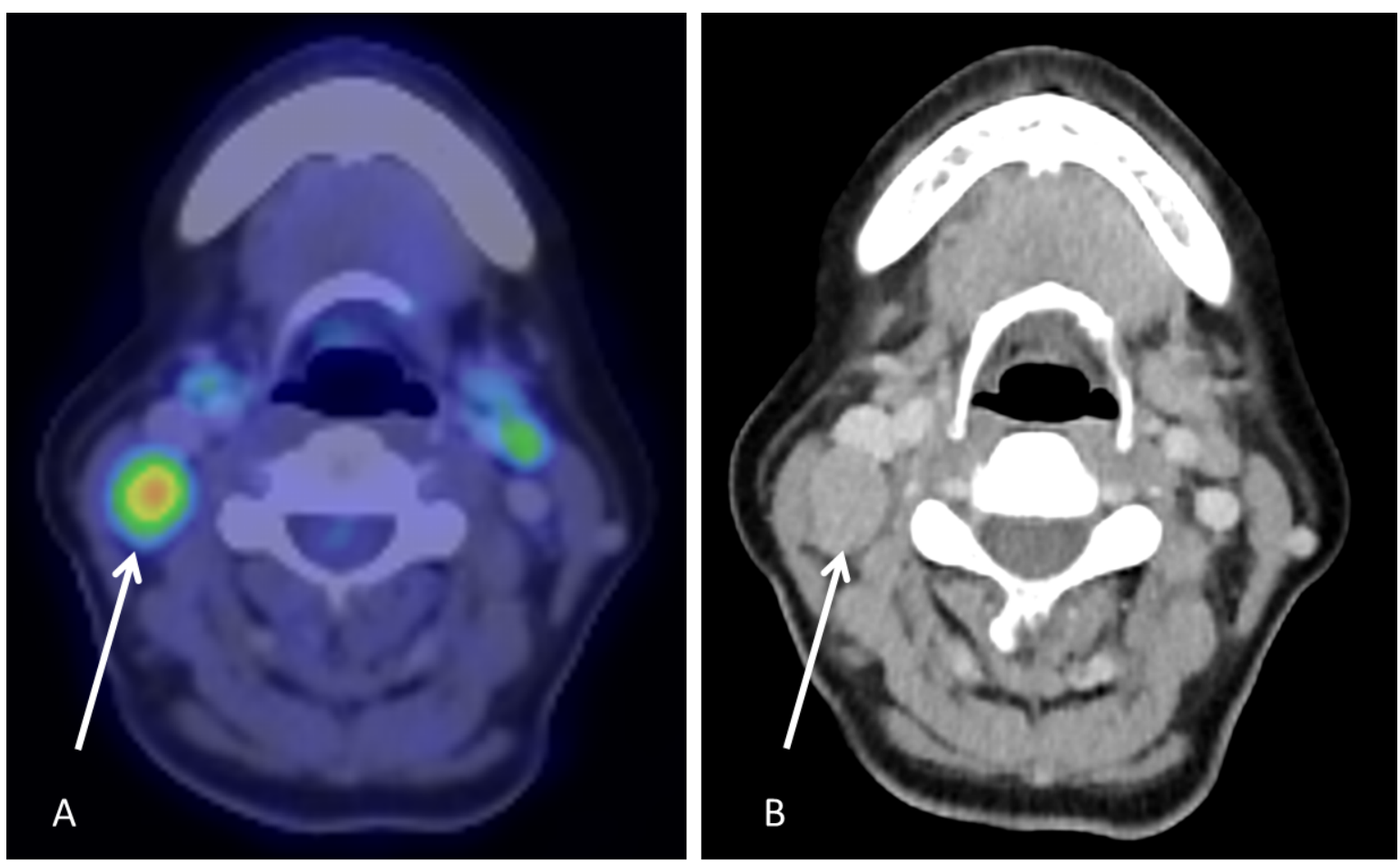

Fig. 4. (A) Positron Emission Tomography and (B) Computed Tomography images show a very FDG avid (SUVmax) 6.9 and enlarged right cervical level $1 \mathrm{~b}$ lymph node, that is suspicious for nodal recurrence.

$55.4 \%$, anal $/$ rectal $23.8 \%$, female genital tract $18.0 \%$, and urinary tract sites $2.8 \% .^{1}$ The nose, paranasal sinuses, and oral cavity are the most common sites of mucosal melanomas in the head and neck. ${ }^{2}$ Primary middle ear mucosal melanoma and eustachian tube melanoma are rare. To date, there are only 21 cases reported, with 10 cases of melanoma arising from the middle ear, and 11 cases arising from the Eustachian tube. ${ }^{3}$ The mean age at diagnosis was 63.7 years old, with a range of 41 to 88 years. 12 patients were female $(57.1 \%)$ and 9 were male $(42.9 \%)$. The most common presenting symptom of Eustachian tube melanoma was hearing loss (81.5\%) and ear fullness $(54.5 \%)$. Nasoendoscopy of all the patients with eustachian tube melanoma revealed a mass at the pharyngeal orifice of the Eustachian tube and $90 \%$ had serous otitis media.

MRI has better soft tissue resolution than $\mathrm{CT}$ and is currently the best imaging technique for Eustachian tube melanomas, giving information on both the primary lesion and cervical lymph nodes. Melanin is paramagnetic and hence, the higher the melanin content in the tumour, the more intense the signal on T1-weighted image, and the lower the signal intensity on T2-weighted image. Amelanotic melanoma will display converse properties with hypointensity on T1-weighted and hyperintensity on T2-weighted images. ${ }^{4}$ However, our patient showed a hypo-enhancing, T1- and T2-weighted isointense lesion even though it was a melanotic melanoma (Figure 2).

The position of the Eustachian tube makes it a challenging area for surgical resection. We combined the maxillary swing and Fisch B infratemporal approaches to excise the tumour and Eustachian tube en-bloc. The Fisch B approach allows good exposure of the carotid artery and this was essential as the preoperative imaging showed close proximity of the tumour to the carotid artery. Hence, exposure of the vessel would allow quick vascular control in the event of inadvertent injury to the vessel.

The role of elective neck dissection for mucosal melanoma of the head and neck is undetermined. ${ }^{5}$ The lymphatic drainage of the Eustachian tube is to the parotid, retropharyngeal and cervical lymph nodes. Bachar et al found regional recurrence rate of $19.7 \% .{ }^{6}$ This significant proportion of recurrence coupled with the aggressive and high mortality rate of recurrent disease, should compel the surgeon to consider an elective neck dissection, especially since the surgery for the primary tumour is a major surgery intended to cure. In our case, the patient subsequently had cervical lymph node metastases, illustrating this point. In particular, level 2 should be addressed in the elective neck dissection. 
Sentinel lymph node biopsy is performed in cutaneous melanoma to determine the regional lymph node status in early stage disease as lymph node involvement is an important prognostic indicator. ${ }^{7}$ Mucosal melanoma is different from cutaneous melanoma, as most patients will develop distant metastases independent of lymph node status. ${ }^{8}$ At present, there is insufficient information on the accuracy or clinical utility of sentinel lymph node biopsy in mucosal melanoma. ${ }^{9}$

The role of radiotherapy in mucosal melanoma of the head and neck is favoured as adjuvant treatment rather than primary therapy. ${ }^{10}$ The mainstay treatment is radical surgical resection and adjuvant radiotherapy in select patients with high-risk features. In spite of multimodality local treatment, the outcomes for mucosal melanoma remain poor. ${ }^{11}$ The 5 -year overall survival of head and neck mucosal melanomas is poor at less than $30 \%$ in most series. ${ }^{12}$ In a review of the 11 cases of Eustachian tube mucosal melanoma available in the literature, only 4 patients had no evidence of disease at the time of reporting. 1 patient died, 3 had distance metastasis, 2 had local recurrence and 1 had partial response. ${ }^{3}$ The follow-up period of the patients with no evidence of disease was low and ranged from 8 to 15 months. With longer follow-up, the prognosis of this condition may be grimmer. Of the 10 cases available in the literature of Eustachian tube melanoma, ours provides the longest follow up available.

Michel et al. compared 3 staging systems for sinonasal mucosal melanomas-Ballantyne/Prasad 2004, American Joint Committee on Cancer (AJCC) mucosal melanoma tumour/node/metastasis (TNM), and AJCC carcinoma TNM. In their retrospective study of 35 patients with sinonasal mucosal melanoma, they found that only the AJCC carcinoma TNM was significantly correlated with overall survival. ${ }^{13}$ We wonder if this finding is applicable to Eustachian tube mucosal melanomas, as although the Eustachian tube is not a direct component of the sinonasal tract, the mucous membrane of the Eustachian tube and sinonasal tract is similar and in continuity. Based on Ballatyne/Prasad staging, our patient's tumour was stage 1 level 2 as the tumour was localized and did not invade the cartilage. ${ }^{14,15}$ Based on the 8th edition AJCC staging of head and neck mucosal melanomas, our patient's tumour was T3N0MO. There is no prognostic stage grouping in AJCC for head and neck mucosal melanomas.

Precision medicine and immunotherapy has a growing role in cancer management. Tumour genome sequencing and molecular pathology are increasingly used to tailor therapy, and immune checkpoint inhibitors has led to long remissions of more than 10 years for advanced malignant melanoma. ${ }^{16}$ Frequently altered genes such as KIT, BRAF, N-RAS and GNAQ, and molecular pathways like PI3K-Akt-mTOR in mucosal melanoma are being studied. Research on the use of c-KIT inhibitors such as imatinib in melanomas with c-KIT alterations is on-going. ${ }^{17}$ However, implementing these advances in research is challenging due to the rarity of head and neck mucosal melanomas, and high cost. ${ }^{18} \mathrm{C}$-kit mutation analysis and BRAF sequencing for our patient did not detect any mutation.

\section{Conclusions}

Mucosal melanoma of the Eustachian tube is a rare condition. Majority of patients present with localised disease, without nodal metastases. Surgical excision is challenging due to the deep-seated nature of the Eustachian tube from the nasopharynx to the middle ear, and close proximity to critical structures such as the internal carotid artery and facial nerve. Although the role of elective neck dissection is undetermined in the literature, we recommend performing based on current limited evidence. Despite multimodality treatment, outcomes remain poor with a high rate of mortality.

\section{REFERENCES}

1. Chang AE, Karnell LH, Menck HR. Menck. The National Cancer Data Base report on cutaneous and noncutaneous melanoma: a summary of 84,836 cases from the past decade. The American College of Surgeons Commission on Cancer and the American Cancer Society. Cancer 1998;83:1664-78.

2. Ascierto PA, Accorona R, Botti G, Farina D, Fossati P, Gatta G, et al. Mucosal melanoma of the head and neck. Crit Rev Oncol Hematol 2017;112:136-152.

3. Maxwell AK, Takeda H, Gubbels SP. Primary Middle Ear Mucosal Melanoma: Case Report and Comprehensive Literature Review of 21 Cases of Primary Middle Ear and Eustachian Tube Melanoma. Ann Otol Rhinol Laryngol 2018;127:856-863.

4. Yang BT, Wang ZC, Xian JF, Chen QH. MR imaging features of primary mucosal melanoma of the eustachian tube: report of 2 cases. AJNR Am J Neuroradiol 2009;30:431-3.

5. Tacastacas JD, Bray J, Cohen YK, Arbesman J, Kim J, Koon HB, et al. Update on primary mucosal melanoma. J Am Acad Dermatol 2014;71:366-75.

6. Bachar G, Loh KS, O'Sullivan B, Goldstein D, Wood S, Brown D, et al. Mucosal melanomas of the head and neck: experience of the Princess Margaret Hospital. Head Neck 2008;30:1325-31.

7. Gonzalez A. Sentinel Lymph Node Biopsy: Past and Present Implications for the Management of Cutaneous Melanoma with Nodal Metastasis. Am J Clin Dermatol 2018;19:24-30.

8. Gavriel H, McArthur G, Sizeland A, Henderson M. Review: mucosal melanoma of the head and neck. Melanoma Res 2011;21:257-66. 
9. Stárek I, Koranda P, Benes P. Sentinel lymph node biopsy: A new perspective in head and neck mucosal melanoma? Melanoma Res 2006;16:423-7.

10. Christopherson K, Malyapa RS, Werning JW, Morris CG, Kirwan J, Mendenhall WM. Radiation therapy for mucosal melanoma of the head and neck. Am J Clin Oncol 2015;38:87-9.

11. Schmidt MQ, David J, Yoshida EJ, Scher K, Mita A, Shiao SL, et al. Predictors of survival in head and neck mucosal melanoma. Oral Oncol 2017;73:36-42.

12. López F, Rodrigo JP, Cardesa A, Triantafyllou A, Devaney KO, Mendenhall WM, et al. Update on primary head and neck mucosal melanoma. Head Neck 2016;38:147-55.

13. Michel J, Perret-Court A, Fakhry N, Braustein D, Monestier S, Richard MA, Grob JJ, et al. Sinonasal mucosal melanomas: the prognostic value of tumor classifications. Head Neck 2014;36:311-6.

14. Ballantyne AJ. Malignant melanoma of the skin of the head and neck. An analysis of 405 cases. Am J Surg 1970;120:425-31.

15. Prasad ML, Patel SG, Huvos AG, Shah JP, Busam KJ. Primary mucosal melanoma of the head and neck: a proposal for microstaging localized, Stage I (lymph node-negative) tumors. Cancer 2004; 100:1657-64.

16. Farid M, Toh HC. World Cancer Day 2019 - Don't Stop Thinking About Tomorrow. Ann Acad Med Singap 2019;48:42-44.
17. Carvajal RD, Antonescu CR, Wolchok JD, Chapman PB, Roman RA, Teitcher J, et al. KIT as a therapeutic target in metastatic melanoma. JAMA 2011;305:2327-34.

18. Chng WJ. Cancer in 2019 - Progress and Challenges - A Perspective. Ann Acad Med Singap 2019;48:45-47.

Vanessa YJ $\underline{\text { Tan, }}{ }^{1}$ MBBS (S'pore), MRCS (Glas), MMed (ORL),

Barrie YB Tan, ${ }^{1} M B B S$ (S'pore), MRCS (Edin), MMed (ORL),

Si Wei Kheok, ${ }^{2} M B B S$, Terence WK $\underline{\text { Tan }},{ }^{3} M B B S$, FRCR, FAMS,

Hiang Khoon Tan, ${ }^{4}$ MBBS (S'pore), FRCS (Edin)

${ }^{1}$ Otorhinolaryngology, Head and Neck Surgery, Singapore General Hospital, Singapore

${ }^{2}$ Department of Diagnostic Radiology, Singapore General Hospital, Singapore

${ }^{3}$ Radiation Oncology, National Cancer Centre, Singapore

${ }^{4}$ SingHealth Duke-NUS Head and Neck Centre, National Cancer Centre, Singapore

Address for Correspondence: Dr Vanessa YJ Tan, Outram Park, Singapore 169608

Email: vanessa.tan.y.j@singhealth.com.sg 